\section{Photosynthetically active radiation - a new unit}

Sir-It is meaningful and useful, and now fashionable in studies involving photosynthesis, to express light intensities as micro-einsteins of photosynthetically active radiation per square metre per second: $\mu \mathrm{E} \mathrm{m}^{-2} \mathrm{~s}^{-1}$ (PAR). This unit is not a standard international one, as is a mole of photons. It could be confusing since to physicists the Einstein number is a velocity relative to that of light. In fact, it is obviously not a purely physical unit but a physico-botanical one, since spectra of photosynthetically active light differ among green plants, red and brown algae, diatoms, cyanophytes and other photosynthetic bacteria according to their respective complements of photosynthetic pigments: chlorophylls, bilin pigments, and so on. It is awkward to type, since it involves negative exponents and a Greek letter found on few typewriters. It is also a long phrase to use in common parlance, and referring to it briefly and colloquially as a "micro-einstein" is wrong.

I therefore suggest that for physicobotanial usage, in such fields as plant physiology, limnology and oceanography, we use a new nominal unit, the albert: $1 \mathrm{alb}$ $=1 \mu \mathrm{E} \mathrm{m} \mathrm{m}^{-2} \mathrm{~s}^{-1}$ (PAR). (This continues to commemorate Albert Einstein.) It is a derived unit, like a joule or a roentgen. Very approximately, one kilo-lux is equivalent to about $20-40$ alb, while full sunlight provides a light intensity in the range of 2,000-4,000 alb (depending on the latitude and on the kind of photosynthetic system involved).

RaLPH A. LewiN Scripps Institute of Oceanography, University of California, San Diego, La Jolla, California 92093, USA

\section{Splicing and the evolution of introns}

SIR-The recent suggestion by CavalierSmith $^{1}$ that introns have evolved by duplicative transposition within eukaryotic genomes is one which we believe deserves serious consideration. We would, however, like to offer an alternative to the model of transposition at the level of DNA. We propose that if introns are indeed the vestiges of transposable elements, they represent a class of retrovirus-like transposons or retrotransposons ${ }^{2}$. The attraction of this latter model is that it gives the splicing mechanism a central role in the evolution of introns. There is a growing body of evidence to suggest that splicing is intron encoded $^{3-6}$. Consequently, it is reasonable to look for evolutionary explanations of the splicing mechanism in the context of the evolution of the introns themselves.

In our view, the ancestral intron genomes were replicated into RNA copies simply because of their insertion within transcriptionally active regions of the host genome. Splicing was necessary not only to minimize negative effects on host gene expression ${ }^{1,7}$ but also, perhaps more importantly, to generate new copies of the intron genome free of flanking exon sequences?

These spliced intron copies were then available for reverse transcription and reinsertion elsewhere in the genome. Most modern introns have probably lost much of their original genetic content and may be considered as degenerate evolutionary relics. An exception is the set of splicing signals which must be retained because of its importance to host cell survival.

Finally, we would like to point out that any particular model of the origin of introns does not argue for or against a subsequent short-term or long-term evolutionary role for these elements within the eukaryotic genome.

\section{Department of Biology,}

Donal A. Hickey

BERnHaRd F. BENKEL

University of Ottawa,

Ottawa, Ontario, Canada

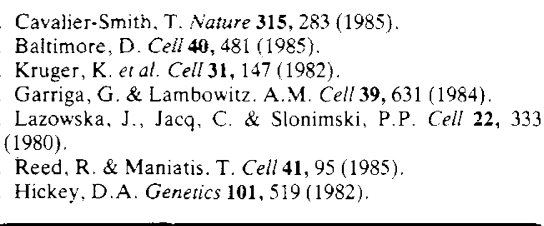

\section{Current density and permittivity}

SIR-John Maddox (Nature 316, 101; 1985), in his article on Maxwell's displacement current should have been more careful with his electrical units. $J$ is not a current but a current density measured in $A$ $\mathrm{m}^{-2}$. The preferred name for $\varepsilon$ is permittivity; it is not a scalar but in general a second rank tensor. The name dielectric constant for $\varepsilon$ should be avoided, as $\varepsilon$ usually varies with temperature and frequency and can vary with applied field strength. (See Quantities, Units and Symbols, Royal Society, London, 1975 and addenda 1981).

Department of Physics,

H. MYKuRA

University of Warwick

Coventry CV4 $7 A L, U K$

\section{Null hypotheses and the misuse of statistics}

SIR-Van Valen pleads for greater freedom for the applied scientist constrained by the "narrow Neyman-Pearson view of statistics as hypothesis-testing" (Nature 314, 230; 1985). He advocates a more flexible use of statistics and although in the main we do not disagree with this, our own experience in medical statistics leads us to believe the problem is more fundamental than misuse of null hypotheses.

Statistics involve three ingredients technical statistics, language and method. Mastery of all three would be ideal. Mastery of technical aspects is a minimum requirement, based on the organization of ideas according to established recipes defined by previously encountered 'typical' research problems. The technical element then is more adapted to provoking action than to reflection, and if we agree that reflection followed by action underlies scientific progress, then we might reasonably consider it perverse for a researcher to be incapable of formulating hypotheses in the absence of stimuli from a computer.

The language of statistics is that of mathematical models and probability theory underlying the calculations. With the advent of computer packages and a much wider access to $P$-values, increasingly solicited by journals, mastery of the language is rapidly becoming seen as a specialist area only of use in unravelling the most complex situations.

Method is concerned with the fundamental approach and not immediate operational questions. Under this heading we have for example Bayesian techniques, parameter estimation and ... hypothesis testing. The mistake is to regard these approaches as fundamental principles and to try and squeeze all problems under one or another heading. Worse still is to try and answer questions using one approach which can only be answered using another. To take epidemiology as an example, we almost have to admire the ingenuity whereby, to avoid modelling, problems are formulated in terms of the most untenable null hypotheses. $\mathrm{Re}$ searchers aware of differential effects and yet with hopelessly inappropriate means for quantifying them find themselves writing $P<0.0001$ to underline an effect of possibly greater importance than one for which, for instance, $P<0.01$. Such an expression can find little motivation or meaning in the asymptotic framework of the distribution theory underlying any test, even when appropriate, and yet the optimistic expression $P<10^{-7}$ is by no means unknown in the medical literature.

To would-be users of statistics it should be made clear that the acceptance or rejection of a null hypothesis does not establish scientific knowledge with the same degree of conviction and this is entirely consistent with Popper's outlook. Unfortunately we are tempted to overlook this fundamental logical asymmetry: thus while some will invoke a non-significant result as evidence for the equivalence of treatments others will invoke a nonsignificant result as proof of the comparability of patient groups before treatment. In contrast, a significant result will almost always be given a weight disproportionate to its scientific import, the constant of disproportionality varying inversely with the level of significance.

Unité de Recherches Statistiques,

INSERM U21,

94807 Villejuif Cedex, France 